\title{
LAW: A Novel Mechanism for Addressing Hidden Terminal Problem in LTE-U and Wi-Fi Networks
}

\author{
Anand M. Baswade, Touheed Atif, Bheemarjuna Reddy Tamma, Antony Franklin A
}

\begin{abstract}
Recently, the use of LTE in unlicensed spectrum (LTE-U) has gained a lot of attention. One of the daunting task before any such employment was to ensure the fair sharing of unlicensed spectrum between LTE-U and Wi-Fi Radio Access Technologies (RATs), which now seems to be well addressed in the literature. However, along with fair sharing, the efficient utilization of unlicensed spectrum is also of profound significance, which pushes the need for coordination between LTE-U and Wi-Fi. Hence, this letter proposes a novel LTE-U and Wi-Fi (LAW) inter-RAT coordination mechanism for a more efficient utilization of the unlicensed spectrum. The aim is to address the inter-RAT hidden terminal problem between LTE-U and Wi$\mathrm{Fi}$ and thereby offer better spectral efficiency. We modify the regular CTS-to-Self frame and suggest transmissions of modified CTS-to-Self from LTE-U nodes to solve this hidden terminal issue. Further gains are extracted by allowing simultaneous transmissions of LTE-U and Wi-Fi whenever possible.
\end{abstract}

Index Terms-Hidden terminal, Wi-Fi, LTE-U, CTS-to-Self.

\section{INTRODUCTION}

Duty cycled LTE-U [1] has been very popular because of the minimal changes it requires in the existing LTE protocol stack, before being deployed. It achieves this by following a discontinuous transmission in the unlicensed spectrum using Carrier Sense Adaptive Transmission (CSAT) [2]. where, LTE-U transmits for some period called as LTE-U ON period and halts for some period termed as the LTE-U OFF period. [1], [2] have shown that these ON and OFF transmissions can fairly coexists with Wi-Fi. However, the inter-RAT (LTE-U and Wi-Fi) hidden terminal problem emerging out of these coexistence have not been considered in the literature. To the best of our knowledge, this is the first proposal for inter-RAT coordination in the hidden terminal scenarios to improve the performance of Wi-Fi and hence-forth utilize the spectrum more efficiently.

Wi-Fi follows Carrier Sense Multiple Access with Collision Avoidance (CSMA/CA) mechanism to access the channel, owing to which a Wi-Fi node considers Clear Channel Assessment (CCA) as busy if either a valid Wi-Fi signal exceeding the Carrier Sense Threshold (CST) $(-82 \mathrm{dBm})$, or any other signal exceeding the Energy Detection Threshold (EDT) (-62 $\mathrm{dBm})$ is received. As a result, we assume that Wi-Fi nodes detect the LTE transmissions with EDT. Depending on the strength of these LTE-U transmissions received by the Wi-Fi AP operating on the same channel, we categorize all the coexistence scenarios into three types as follows:

1. Inside EDT: Wi-Fi AP is inside the EDT range of LTE-U eNB. In this scenario, both the AP and LTE-U share the chan-

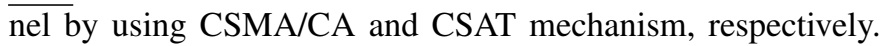
And as a result, any transmission from LTE-U eNB prevents the AP from accessing the channel. Hence, either AP or eNB can successfully transmit at any given time. However, all Wi-Fi users perform fairly because AP can serve them only in LTE-U OFF period.

2. In-between EDT and CST: Wi-Fi AP is outside EDT but inside CST range of LTE-U eNB. In this scenario, AP can transmit to some of its users even in LTE-U ON period as its CCA mechanism detects channel as free for these users. This results in an inter-RAT hidden terminal problem, as the users outside the EDT range of LTE-U and experiencing more than minimum required SINR for transmission can access the medium and also can be served the AP, while the ones inside the EDT can neither access the channel nor the AP can serve them. Hence, we classify the users based on above criterion as non-victim and victim respectively

3. Outside CST: Wi-Fi AP is outside CST range of LTE-U eNB. Again, in this scenario both eNB and AP can transmit simultaneously and hence the Wi-Fi users will again be divided as victim and non-victim users.

To solve such problems, involving an overlap of two cells belonging to the same RAT, Inter-Cell Interference Coordination (ICIC), enhanced-ICIC (eICIC) for LTE and Request-ToSend (RTS) and Clear-To-Send (CTS) handshake for Wi-Fi are well studied. However, the scenario presented above involves two different RATs and straightforward extension from the above techniques cannot be employed. Therefore, in this letter, we propose a novel LTE-U And Wi-Fi (LAW) coexistence mechanism, aiming to improve the overall performance. Our approach uses Self-CTS/CTS-to-Self mechanism of Wi-Fi embedded with an additional information, without significant modification. This is achieved by using the previously available reserved fields.

In [3] and [4], the authors proposed transmission of SelfCTS directly from LTE-U eNB to inform the Wi-Fi AP about its presence and showed significant performance gain in the hidden terminal scenario. However, sending Self-CTS from LTE-U eNB fails when Wi-Fi AP cannot receive Self-CTS (i.e., AP is outside CST range) and thus the performance remains similar to Standard Wi-Fi. We refer to the performance of Wi-Fi in the absence of Self-CTS from LTE-U eNB as Standard Wi-Fi (SW) scheme, and in the presence of Self-CTS directly from LTE-U eNB as LCTS scheme. This can be solved up to some extent by transmitting Self-CTS from UE instead of eNB. In [3], the authors also proposed transmission of SelfCTS through LTE-U UE, we call this scheme as UE-CTS. Nonetheless, in both LCTS and UE-CTS schemes, the regular Self-CTS messages are sent from LTE-U which Wi-Fi uses extensively to address hidden terminal problem among Wi-Fi 
TABLE I: Duration/ID field encoding [5] with suggested modification

\begin{tabular}{|c|c|c|c|}
\hline Bits 0-13 & Bit 14 & Bit 15 & Usage \\
\hline \multicolumn{2}{|c|}{$0-32767$} & 0 & Duration value $(\mu \mathrm{s})$ \\
\hline 0 (all) & 0 & 1 & Fixed value in PCF during CFP \\
\hline $\mathbf{1}$ & $\mathbf{0}$ & $\mathbf{1}$ & When LTE-U is ON \\
\hline $\mathbf{2}$ & $\mathbf{0}$ & $\mathbf{1}$ & When LTE-U is OFF \\
\hline $3-16383$ & 0 & 1 & Reserved \\
\hline
\end{tabular}

nodes. Therefore, due to the transmission of the regular SelfCTS message by LTE-U, the Wi-Fi AP cannot intelligently decide upon whom to serve and utilize the unlicensed spectrum most efficiently.

\section{PROPOSED MECHANISM}

The fundamental problem with the operation of different RATs on the same channel is that the lack of communication/coordination leads to collisions or interference resulting in poor utilization of the unlicensed spectrum. The proposed LAW mechanism makes efficient use of the Self-CTS frame of Wi-Fi to address the inter-RAT hidden terminal problem described in the previous section. The Self-CTS frame consists of the Duration/ID field which informs neighboring Wi-Fi devices to defer their channel access for a duration by setting Network Allocation Vector (NAV) value. The Duration field is 16-bit and has many reserved values as shown in Table I. We used two values from these reserved values to enable interRAT coordination. LTE-U eNB informs one of its users (also called as an agent) through the licensed spectrum to send Self-CTS through its Wi-Fi interface. The value 32769 is sent by LTE-U when it is about to use unlicensed band for its transmission (i.e., LTE-U is ON) and the value 32770 is sent by LTE-U when it stops using unlicensed band (i.e., LTE-U is OFF). Once Wi-Fi AP receives the Self-CTS of the LTE-U agent, it behaves as follows: If it is LTE-U ON period, then Wi-Fi will serve only the non-victim (NV) users. Whereas, if it is LTE-U OFF period, then Wi-Fi will first serve only the victim $(\mathrm{V})$ users for a specified duration (called $V_{\text {time }}$ ) and then continues serving all of the users.

Workflow of the proposed LAW Mechanism: The flow of events is shown in Fig. 1. LTE-U follows ON-OFF cycles to fairly share the channel with Wi-Fi. It informs ON-OFF information to Wi-Fi AP through one of its users (agent). The agent then sends the modified Self-CTS through its Wi-Fi interface after PCF (Point Coordination Function) Interframe Space (PIFS) duration with one of the above mentioned reserved values. We urge transmitting the modified Self-CTS frame after PIFS duration to ensure that LTE-U agent will occupy the channel earlier than any Wi-Fi node. Once LTE-U eNB informs the LTE-U agent to send Self-CTS, the LTE-U

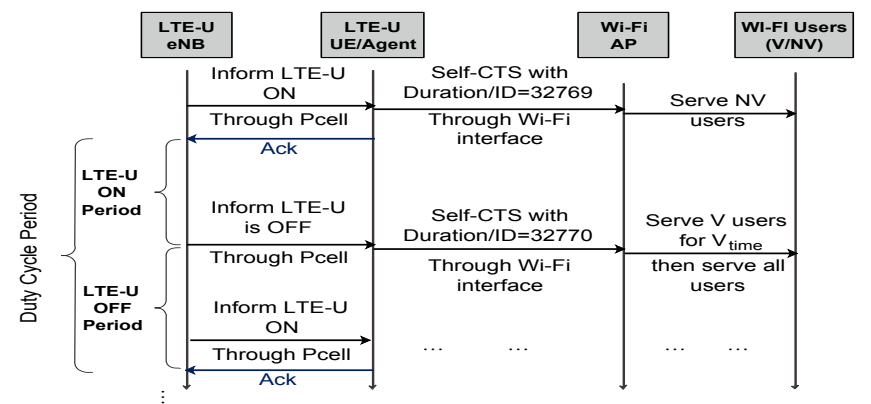

Fig. 1: Flow diagram for the proposed LAW mechanism. agent sends the Self-CTS through its Wi-Fi interface and sends an acknowledgment (ACK) back to LTE-U eNB through the licensed spectrum.

1) Time to serve only the victim users: If the entire LTE-U OFF period is used to serve the victim users, the result can be, victim users gaining an undue advantage in terms of throughput. To avoid this, we find the minimum $V_{\text {time }}$ duration within LTE-U OFF period to serve the victim users, thereby ensuring throughput fairness among the Wi-Fi users (both victim and non-victim users). Variation in $V_{\text {time }}$ can control how much perquisite victim users have over the nonvictim users. When $V_{\text {time }}$ is set to zero, the LAW mechanism degenerates to DCF mechanism. Any value of $V_{\text {time }}$ between 0 and LTE-U OFF period will give better throughput for the victim users (if present) as well as for the entire Wi-Fi network. Our approach to realize optimum $V_{\text {time }}$ duration is to adjust $V_{\text {time }}$ by observing the throughputs of users in the previous duty cycle period. The updated $V_{\text {time }}$ in terms of previous $V_{\text {time }}^{\text {old }}$ duration can be given as:

$$
V_{\text {time }}=\min \left(\frac{R_{n v}^{n e w}}{R_{v}^{n e w}} \cdot V_{\text {time }}^{\text {old }}, \mathrm{LTE}-\mathrm{U} \_\mathrm{OFF} \_ \text {Period }\right) \text {. }
$$

$R_{n v}^{n e w}$ and $R_{v}^{\text {new }}$ are calculated using exponential moving average of throughputs of $\mathrm{NV}$ and $\mathrm{V}$ users, respectively as:

$$
R_{x}^{\text {new }}=(1-\alpha) R_{x}^{\text {curr }}+\alpha R_{x}^{\text {old }} \quad x \in\{v, n v\},
$$

Where $R_{x}^{\text {curr }}$ is average instantaneous throughput, $R_{x}^{\text {old }}$ is the past throughput, and $\alpha$ is an exponential smoothing parameter. The idea behind using a parameter for exponential smoothing is to employ the history to ensure throughput fairness in long-term. This means that throughput unfairness caused in a particular duty cycle is carried to subsequent duty cycles, to achieve a long-term fairness. As a result, deciding $V_{\text {time }}$ using a memory based version of $R_{v}$ and $R_{n v}$ would provide long-term fairness among the users.

Furthermore, we provide the following intuitive explanation for using Eqn. (1). If the ratio of average non-victim to average victim throughput is more than one, we seek to increase $V_{\text {time }}$, and vice-versa. And, to achieve this we multiply the above ratio (i.e., $R_{n v} / R_{v}$ ) by the previous $V_{\text {time }}$. This ensures that we get the desired variation (equivalent to the above explanation) while incorporating a feedback mechanism (using $R_{v}$ and $R_{n v}$ ) and simultaneously being self-sufficient. In addition, its simplicity can be easily incorporated into practical systems.

2) Which LTE-U UE to choose as an agent?: All the active UEs which are under the influence of Wi-Fi AP (i.e., those which can receive the beacons of Wi-Fi AP through their Wi-Fi interface) report the SSID of Wi-Fi AP to the eNB over the licensed channel. The UEs get SSID from beacons of AP (to listen to beacons user does not require an association with the AP). Now, LTE-U eNB chooses a user which has the highest Received Signal Strength Indicator (RSSI) in the unlicensed channel as an agent to send out the modified Self-CTS. If multiple APs are overlapping with the LTE-U eNB, the LTE-U eNB selects one user for each SSID as an agent.

$$
\text { Agent }_{i}=\max \left(R S S I_{i}\right) \quad \forall i \in\{S S I D\} .
$$


3) Minimizing the inter-RAT signaling overhead: The LTE-U agent sends a Self-CTS only when there is a change in LTE-U ON and OFF periods. And the Wi-Fi AP keeps track of the ON and OFF periods to serve its users accordingly during LTE-U ON-OFF cycles.

4) How does AP know victim and non-victim users?: Once AP knows the presence of LTE-U eNB because of modified Self-CTS, it starts observing the throughput of its users. Users getting low or zero throughput only during the ON period and not in OFF period are classified as victim users, and rest as non-victim users.

5) What if there are multiple Wi-Fi networks?: LTE-U CSAT adaptively chooses ON period based on neighboring Wi-Fi networks. So, when LTE-U agent transmits the SelfCTS, all the neighboring Wi-Fi networks will receive it, which in turn share the channel by following CSMA/CA in the ONOFF cycle to serve their victim and non-victim users.

6) Effect of Wi-Fi on LTE-U: LTE-U operates in both licensed and unlicensed spectrum whereas Wi-Fi operates only in unlicensed spectrum. In LTE-U ON period, LTE-U UEs may get inter-RAT interference from neighbor Wi-Fi networks. But, LTE-U eNB can serve its affected users using licensed spectrum and as a recompense can provide more resources to the unaffected users in the unlicensed spectrum which is not possible for Wi-Fi AP. Hence, we have done classification of victim and non-victim users only for Wi-Fi networks.

\section{Performance Evaluation}

To study the performance of Wi-Fi users in the presence of LTE-U network, we consider three scenarios as discussed in Section I. An LTE-U eNB and a Wi-Fi AP are deployed $50 \mathrm{~m}$ (outside CST), 35m (in-between EDT and CST), and $10 \mathrm{~m}$ (inside EDT) apart as shown in Fig 2. STA1 and STA2 are two Wi-Fi users with STA1 being a victim user and STA2 being a non-victim user. The simulation parameters are as given in Table II. LTE-U eNB is following a 50\% duty cycle with a period of $20 \mathrm{~ms}$, thereby engendering a $10 \mathrm{~ms}$ ON period followed by a $10 \mathrm{~ms}$ OFF period. The performance of proposed LAW scheme is compared with existing SW, LCTS, and UE-CTS schemes discussed in Section I. According to [6], Downlink (DL) dominates with $80-90 \%$ of data traffic while Uplink (UL) contributes only $20-10 \%$. Hence, along with UL+DL traffic scenarios results are also shown for DL only traffic scenarios.

The throughput results of Wi-Fi network are shown in Fig. 3 for DL traffic scenario. It can be observed that the proposed LAW scheme outperforms all the schemes as it adds intelligence to Wi-Fi AP in deciding which user to serve based on LTE-U ON and OFF periods.

To delineate the improvements in these three scenarios we discuss them individually, covering all the aspects.

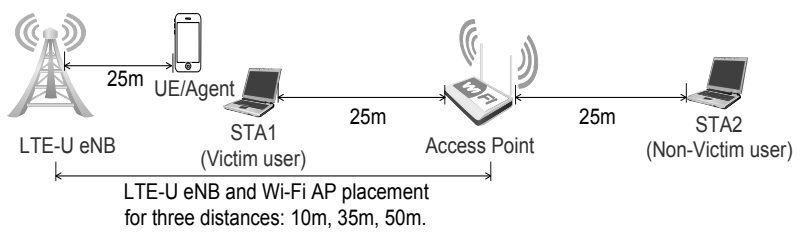

Fig. 2: LTE-U and Wi-Fi hidden terminal scenario.
TABLE II: Wi-Fi \& LTE-U Parameters

\begin{tabular}{|c|c|c|c|}
\hline \multicolumn{2}{|c|}{ Wi-Fi parameters } & Common Parameters \\
\hline Parameter & Value & Parameter & Value \\
\hline$C W_{\min }, C W_{\max }$ & 16,1024 & Tx Power & $20 \mathrm{dBm}$ \\
\hline PHY, MAC Header & 128,272 bits & Operating Freq. & $5.3 \mathrm{GHz}$ \\
\hline ACK, RTS & 240,288 bits & Noise & $-101 \mathrm{dBm}$ \\
\hline Payload, MPDU & 8148 bits, 4 & Bandwidth & $20 \mathrm{MHz}$ \\
\hline Slottime,CTStimeout & $9,50 \mu \mathrm{s}$ & Antenna Ht. & 10 meter \\
\hline DIFS, SIFS & $34,16 \mu \mathrm{s}$ & User Antenna Ht. & 1 meter \\
\hline Beacon Interval, $\alpha$ & $100 \mathrm{~ms}, 0.5$ & Traffic & UDP \\
\hline \multicolumn{2}{|c|}{ Parameter } & \multicolumn{3}{|c|}{ Value } \\
\hline Wi-Fi PHY Rates (Mbps) & $13,26,39,52,78,104,117,130$ \\
\hline Required SNR (in dB) & $5,7,9,13,17,20,22,23$ \\
\hline Path Loss Model [7] & $36.7 \log 10(\mathrm{~d}[\mathrm{~m}])+22.7+26 \log 10($ freq[GHz]) \\
\hline
\end{tabular}

1. Inside EDT:- In this scenario, the AP can transmit only during LTE-U OFF period, thereby hindering any scope for improvement. We believe that the inside EDT scenario is rare as both LTE-U eNB and AP try to select a channel which is having very low interference levels (i.e., possibly used by far placed AP or eNB) and hence we do not consider it further.

2. In-between EDT and CST:- When the Wi-Fi AP is outside EDT and inside CST, (i.e., in-between scenario), LCTS, UE-CTS and proposed LAW schemes can detect the presence of LTE-U, whereas SW scheme cannot. This causes the SW AP to transmit to the victim users during the LTE-U ON period, incurring huge transmission losses. On the other hand, LCTS and UE-CTS on detecting the presence of LTE-U behaves very conservatively and transmits packets only during the LTE-U OFF period. As a result, the channel during the entire ON period gets wasted, while the AP and the non-victim users perhaps could have successfully communicated using lower modulation and coding schemes. LAW successfully exploits this fact by transmitting only to the non-victim users during the ON period and later to all of its users during the OFF period, thereby avoiding retransmission losses as well as gaining the advantage of simultaneous transmissions by LTE-U and Wi-Fi.

3. Outside CST:- When the AP is outside the CST range with respect to LTE-U eNB, the AP in the SW and LCTS schemes cannot detect the presence of LTE-U transmissions, thus making them transmit to the victim users during the ON period. As a result, abundant packet losses occur, causing immense channel wastage. On the other hand, UE-CTS and proposed LAW can detect the LTE-U presence. UE-CTS serves the users only in LTE-U OFF period whereas the proposed LAW scheme with the help of modified Self-CTS serves the users intelligently in both LTE-U ON and OFF periods. This helps the LAW scheme to achieve $80 \%$ more throughput than SW and LCTS schemes as shown in Fig. 3.

Apart from all the throughput gains, the proposed scheme also ensures fairness among the Wi-Fi users. Fig. 4 shows the achieved throughput fairness among the users employing the proposed LAW scheme. Furthermore, both victim and nonvictim users' performance has drastically improved and most importantly the victim user is not victim anymore, having achieved a performance comparable to the non-victim user.

With the introduction of UL traffic in the Wi-Fi network, new challenges occur, with the most prominent one being the imbalance in the UL and DL throughputs of the network. Here, when the AP dumbly serves both the victim and non- 


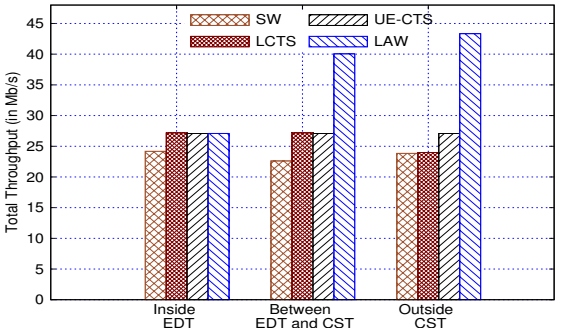

Fig. 3: Total throughput for DL only traffic case. victim users during the LTE-U ON period, the packet losses to victim users causes an exponential increase in the Contention Window (CW) of the AP. Non-victim users, with a full buffer UL traffic and low $\mathrm{CW}$ size, exploit the channel by accessing it recurrently. This leaves the AP with a lower chance to decrement its higher BackOff (BO) value (selected because of increased CW size) and finally access the channel. Hence, DL throughput is lower compared to UL throughput, which is clearly seen in Fig. 5. However, with the proposed LAW scheme, the AP intelligently schedules the users (by deferring from transmitting to the victim user in the LTE-U ON period), preventing the packet losses because of victim users and avoiding the exponential increase in its $\mathrm{CW}$. This allows the AP to maintain its channel accessibility identical to that of the non-victim user and hence improves DL throughput in the Wi-Fi network. Finally, the sum of UL+DL throughput is higher in LAW compared to LCTS and SW schemes, making it the most efficient scheme.

Multiple users scenario with varying UL traffic:- To obtain the average network behavior, we conducted experiments for 100 seeds and in each seed 10 users are placed uniformly at random in a circle of radius $50 \mathrm{~m}$ from the Wi-Fi AP. We

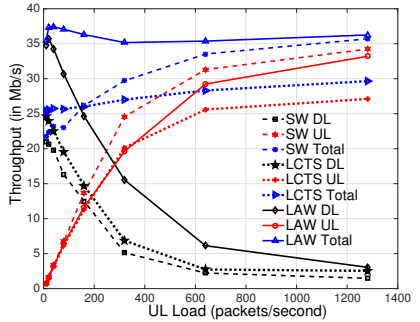

(a) In-between scenario (b) Outside scenario

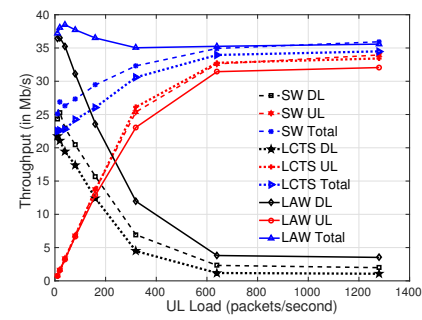

Fig. 6: Wi-Fi network throughput with varying UL load in the networks.

varied UL traffic load of each user from 10 packets/sec to 1280 packets/sec and measured the total throughput of the Wi-Fi network. The average number of victim users observed over 100 seeds are $45 \%$ and $22 \%$ for in-between and outside scenarios, respectively. Figs. $6 \mathrm{a}$ and $6 \mathrm{~b}$ show the average network throughputs for 100 seeds with varying UL traffic. The proposed LAW scheme outperforms both the schemes as AP intelligently serves victim and non-victim users. When UL load is lower, the throughput gain of the proposed scheme is higher, and it reduces as UL load increases because the channel gets shared among all Wi-Fi users and AP. Hence, the LAW scheme has more benefit when there is more DL traffic, and it achieves better throughput compared to other two schemes as shown in Figs. 6a and 6b. When UL load increases, the total throughput improvement over other schemes goes down, but

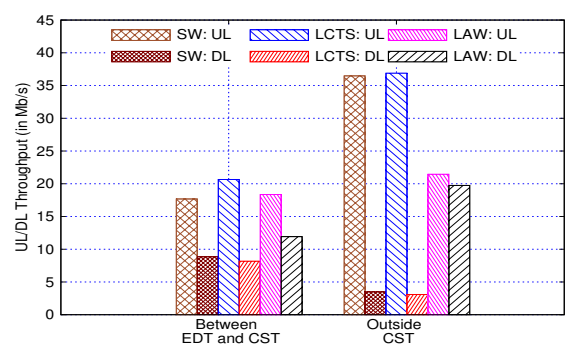

Fig. 5: UL/DL throughputs for UL+DL traffic case. DL throughput is always greater than that of SW and LCTS schemes. Hence, our proposed LAW scheme gives more gain compared to SW and LCTS schemes when DL load is higher, and UL load is lower.

Varying the percentage of victim users in the networks:Figs. $7 \mathrm{a}$ and $7 \mathrm{~b}$ show the results over varying the percentage of victim users in the network for DL only traffic case. Here, LCTS in the in-between scenario and UE-CTS in both the scenarios being aware of LTE-U operates only in LTE-U OFF period. Whereas, SW in the in-between scenario and both SW and LCTS in the outside scenario being unaware of the presence of LTE-U, suffer from transmissions losses and hence show a throughput degradation with increasing percentage of victim users. On the other hand, the LAW scheme remains robust to such an increase and performs substantially better than the other schemes.

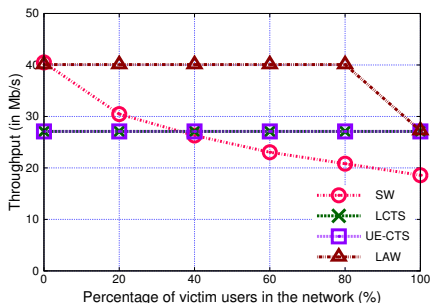

(a) In-between scenario

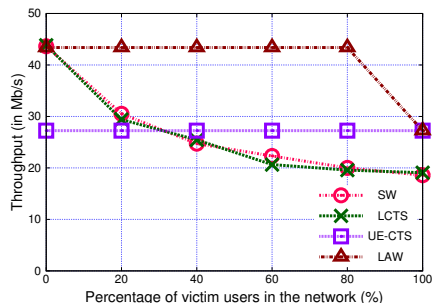

(b) Outside scenario
Fig. 7: Wi-Fi throughput with varying percentage of victim users.

\section{CONCLUSIONS}

This is the first proposal to address $\mathrm{Wi}-\mathrm{Fi}$ performance degradation due to hidden LTE-U eNB i.e., inter-RAT hidden terminal problem. The proposed inter-RAT mechanism, LAW, achieves $80 \%$ higher throughput than that of SW and LCTS schemes in the DL only case. Our results showed that adapting the proposed LAW scheme in the next generation of $\mathrm{Wi}-\mathrm{Fi}$ helps to boost its throughput in the presence of LTE-U or any other similar technology following ON-OFF cycles.

\section{REFERENCES}

[1] "LTE-U SDL Coexistence Specifications." LTE-U Forum, http://www. lteuforum.org/documents.html, 2015.

[2] "LTE in Unlicensed Spectrum: Harmonious Coexistence with Wi-Fi." Qualcomm White Paper, June 2014.

[3] J. Jeon, H. Niu, Q. C. Li, A. Papathanassiou, and G. Wu, "LTE in the Unlicensed Spectrum: Evaluating Coexistence Mechanisms," in Globecom Workshops (GC Wkshps), 2014, pp. 740-745, IEEE, 2014.

[4] E. Chai, K. Sundaresan, M. A. Khojastepour, and S. Rangarajan, "LTE in Unlicensed Spectrum: Are We There Yet?,' in Proceedings of the 22nd Annual International Conference on Mobile Computing and Networking, pp. 135 - 148, Mobicom ACM, October 2016.

[5] I. S. Association et al., "802.11-2012-IEEE Standard Part 11: Wireless LAN MAC and PHY Specifications," Retrived from http://standards. ieee. org/about/get/802/802.11. html, 2012. 
[6] "IMT Traffic Estimates for the Years 2020 to 2030." Report ITU-R M.2370-0, https://www.itu.int/dms_pub/itu-r/opb/rep/R-REP-M. 2370-2015-PDF-E.pdf, July 2015.

[7] 3GPP, "3GPP-TSG-RAN-WG1; Evolved Universal Terrestrial Radio Access (E-UTRA),” Tech. Rep. TR 36.814 V9.0.0, March 2010. 